Title: Acoustic Radiation From Rotating Blades- The Kirchhoff Method in Aeroacoustics

Short Title: The Kirchhoff Method in Aeroacoustics

Author: F. Farassat

Address: Mail Stop 461, NASA Langley Research Center, Hampton, Virginia, 23681-2199

No. of pages: 26 pages (text)

No. of figures: 2 


\title{
Acoustic Radiation From Rotating Blades- The Kirchhoff Method in Aeroacoustics
}

\author{
F. Farassat \\ NASA Langley Research Center, Hampton, Virginia
}

\begin{abstract}
This paper reviews the current status of discrete frequency noise prediction for rotating blade machinery in the time domain. There are two major approaches both of which can be classified as the Kirchhoff method. These methods depend on the solution of two linear wave equations called the $\mathrm{K}$ and FW-H equations. The solutions of these equations for subsonic and supersonic surfaces are discussed and some important results of the research in the past years are presented. This paper is analytical in nature and emphasizes the work of the author and coworkers at NASA Langley Research Center.
\end{abstract}




\subsection{Introduction}

Having obtained a Ph.D. from Cornell University in 1973 under the supervision of Professor William R. Sears, I started to work at NASA Langley Research Center as a visiting scientist of The George Washington University Joint Institute. My task at Langley was to work on the prediction of the noise of helicopter rotors and propellers. We had a good experimental group of engineers collecting helicopter rotor and propeller noise data. However, the capability to predict the noise of these rotating machinery in the design stage to meet community and military noise standards was lacking. By the early Seventies, noise generation mechanisms of helicopter rotors and propellers were understood fairly well. Mainframe computer technology was developing fast. Computer memory size and speed were increasing by leaps and bounds. At that time, propeller noise prediction used Gutin's formula [1] which was of 1930's vintage. Helicopter noise prediction was more advanced and was based on Lowson's formula [2]. Lowson and coworkers had developed helicopter noise prediction codes based on his theory. He had shown the consistency of his result with that of Gutin. Both these formulas are for rotating compact dipoles predicting the loading noise only. Thickness noise prediction was ignored because there was a feeling among acousticians that it was not important for propellers and helicopter rotors. This was contrary to the conclusion of Ernsthausen in Germany [3] and Deming in the U.S. [4] in the Thirties who investigated thickness noise. In fact, Deming had derived a thickness noise formula following the analytic procedure of Gutin but based on Rayleigh's piston in the wall analysis. He also presented numerical results supporting experimental data on the importance of thickness noise at high blade tip speeds.

In the early Seventies, most noise prediction methods were in the frequency domain. This was the natural approach for the researchers because time domain methods generally require powerful computers. By suitable approximation and ingenuity, closed form solutions were obtained for some significant problems using the frequency domain analysis. Aeroacoustics was an active research area and many experimental and theoretical works were being published then. Lighthill's theory was extended to flows in the presence of moving surfaces by Ffowcs Williams and Hawkings in a paper in the Philo- 
sophical Transactions [5]. I had struggled to understand this highly abstract paper and to use it in my Ph.D. thesis. My results were not suitable for immediate application in noise prediction. As I was searching for a direction of research, I was wondering why time domain methods were not used extensively in aeroacoustics. It was in the Summer of 1973 that I met Phil Doak at Langley who was visiting us. I was impressed by his informality and friendliness. I asked him about the lack of interest in the time domain method in aeroacoustics. His response still rings in my ears: "force of tradition"! He then told me that I should ignore the tradition and explore the time domain method for rotating blade noise prediction. It has been an exciting journey and I owe it all to Phil. It is with great pleasure and honor that I dedicate this paper to Phil on the occasion of his eightieth birthday.

Phil's advice could not have been given at a more auspicious time. In addition to the availability of powerful computers, there were also other new tools becoming available to help in advancement of the time domain method. Computational fluid dynamics and more versatile measuring instruments are two of these new tools. One which is perhaps overlooked, and in my opinion the most important, is the analytic tool. The progress since the Fifties in solving partial differential equations, particularly the use of the theory of distributions or generalized functions [6-8], made it possible to get closed form solution of the wave equation with sources on a moving surface in various forms suitable for noise prediction. Ffowcs Williams and Hawkings had used generalized function theory in their paper [5]. The power of this theory is such that much of what was known before such as the Kirchhoff formula for radiation [9], the Lowson's and Curle's formulas [2, 10], can be derived easily and extended. Other questions such as the importance of shock waves in noise generation can naturally be answered using this theory. However, the areas of mathematics applicable in aeroacoustics of moving bodies which also include general tensor analysis and differential geometry are not part of the curriculum of graduate level engineering. Goldstein has used basic generalized function theory to derive some of the important results of aeroacoustics [11]. The present author has published two reports which cover the details of the mathematics used for the wave equation with sources on a moving surface [12-13]. These reports together with Goldstein's book are recommended to the readers to follow the analytic steps below.

In this paper, we review the Kirchhoff method in aeroacoustics using the time domain method. We will emphasize the mathematical aspects of the method. 
Our purpose is to gather the results scattered in numerous technical papers of the author and the coworkers in one place to help those entering the field and the other acousticians interested in rotating blade noise. Recently, it has been possible to predict the quadrupole noise of helicopter rotors in reasonable time on a desktop workstation. The results of some research on high speed impulsive rotor noise are reviewed. We give in detail the derivation of the formulation used to calculate the noise from sources on an open surface (a panel) moving supersonically. The Ffowcs Williams and Hawkings approach [5] can be classified as a Kirchhoff method. In fact, these authors call one of their result as the extension of the Kirchhoff formula to a moving surface. We begin with two linear wave equations called the Kirchhoff $(\mathrm{K})$ and the Ffowcs Williams-Hawkings (FW-H) equations. The solution of the K equation gives the Kirchhoff formula for moving surfaces. We mention the relation of the $\mathrm{K}$ equation to the Ffowcs Williams-Hawkings $(\mathrm{FW}-\mathrm{H})$ equation in a remark that follows. We then discuss how we obtain the solution of several wave equations with various kinds of inhomogeneous source terms appearing in the governing equations. We address the subsonic and supersonic cases separately because they require different treatment.

The organization of the paper is as follows. In the next section we present the governing equations of the Kirchhoff method. Here we give two equations called the $\mathrm{K}$ and the FW-H equations. We recognize four inhomogeneous source terms in these equations. In Section 3, we briefly indicate how the solutions to the wave equations with these source terms can be obtained. We review some of the recent results of quadrupole noise prediction of rotors. A new method to derive a formula suitable for prediction of the noise from a supersonic panel will be presented here. Concluding remarks follow in Section 4.

\subsection{The Governing Equations (K and FW-H)}

The classical Kirchhoff formula [9] describes the solution of the wave equation in the exterior of a surface $S$ as a surface integral over $S$ whose integrand depends on the values of the unknown function, its normal and time derivatives on $S$. It was originally obtained by selecting one of the two functions in the Green's identity in four dimensions [11] as the fundamental solution of the wave equation. The same result can be obtained by an elegant method which requires a more abstract reasoning. Extend the unknown func- 
tion, say the acoustic pressure, to the interior of the surface by assuming that its value is zero there. Now apply the wave operator to this discontinuous function treating all the derivatives as generalized derivatives $[6-8,12,13]$. There will now be inhomogeneous source terms on the right of the wave equation which involve the Dirac delta function with its support on the surface $S$. This inhomogeneous wave equation is what we call the Kequation. Using the Green's function of the wave equation in the unbounded space to formally solve the K equation will give the Kirchhoff formula. One is not restricted to a stationary data surface in this approach. This is precisely the method that Farassat and Myers used to derive the Kirchhoff formula for a moving surface $[13,14]$. The same idea of extending all fluid parameters to the interior of the surface $S$ was utilized by Ffowcs Williams and Hawkings to get the FW-H equation. They used the mass and momentum conservation laws treating all the derivatives as generalized derivatives and manipulated these two equations as Lighthill did to obtain a wave equation $[5,8,11,12]$. Note that in deriving the K equation, we assume that the unknown function satisfies the linear wave equation everywhere while the FW-H equation utilizes the conservation laws. This explains the difference in behavior of the solutions of these equations in the near field. See item 1 of the remarks below.

Let the moving surface be described by $f(\boldsymbol{x}, t)=0$ such that $f>0$ outside the surface and $|\nabla f|=1$ on the surface. Let $p^{\prime}$ be the acoustic pressure. Then the K equation is [12-14]:

$$
\square^{2} \tilde{p}^{\prime}=-\left(p_{n}^{\prime}+\frac{1}{c} M_{n} p_{t}^{\prime}\right) \delta(f)-\frac{1}{c} \frac{\partial}{\partial t}\left[M_{n} p^{\prime} \delta(f)\right]-\nabla \cdot\left[p^{\prime} \boldsymbol{n} \delta(f)\right]
$$

where

$$
\tilde{p^{\prime}}=\left\{\begin{array}{l}
p^{\prime} \text { outside } f=0 \\
0 \quad \text { inside } f=0
\end{array},\right.
$$

$p_{n}^{\prime}$ and $p_{t}^{\prime}$ are the normal and time derivatives of $p^{\prime}$, respectively, $M_{n}$ is the local normal Mach number of the surface, $c$ is the speed of sound and $\boldsymbol{n}=\nabla f$ is the unit outward pointing normal to the surface. The solution of the K equation is the Kirchhoff formula for the moving surface $f=0$ [1214]. 
Next let $f=0$ be a surface which allows the fluid to flow through, assuming again that $f>0$ outside the surface and $|\nabla f|=1$ on the surface. The FW-H equation is $[5,8,12,15]$ :

$$
\begin{aligned}
\square^{2} p^{\prime}= & \frac{\partial}{\partial t}\left\{\left[\rho u_{n}-\left(\rho-\rho_{0}\right) v_{n}\right] \delta(f)\right\} \\
& -\frac{\partial}{\partial x_{i}}\left\{\left[\rho\left(u_{n}-v_{n}\right) u_{i}+p n_{i}\right] \delta(f)\right\} \\
& +\frac{\bar{\partial}^{2}}{\partial x_{i} \partial x_{j}}\left[T_{i j} H(f)\right]
\end{aligned}
$$

where $p^{\prime}$ is $\left(\rho-\rho_{0}\right) c^{2}, \rho$ is the density, and $\rho_{0}$ and $c$ are the density and speed of sound of undisturbed medium. The local normal fluid and surface velocities are denoted by $u_{n}$ and $v_{n}$, respectively. The Lighthill stress tensor is denoted $T_{i j}$ and $\mathrm{p}$ is the surface pressure on $f=0$. Note the above description of the surface $f$ implies that $\nabla f=\boldsymbol{n}$ where $\boldsymbol{n}$ is the unit outward normal to this surface. The Heaviside function is denoted $H(f)$. The bar over the partial differentiation denotes generalized differentiation [6-8, 12]. This notation is used in this paper only when there is the possibility of confusion with ordinary differentiation. We have followed the tradition of using the notation $p^{\prime}$ in Eq. (3) which is defined as follows:

$$
p^{\prime}= \begin{cases}p^{\prime} & \text { outside } f=0 \\ 0 & \text { inside } f=0\end{cases}
$$

Also note that $p$ on the right of Eq. (3) is the gage pressure $p-p_{0}$. It was Ffowcs Williams who proposed that a penetrable moving surface $f=0$ could be more suitable for noise prediction [15, Chap. 11, Sec. 10]. Recent applications have proven him right.

It is evident that we have essentially four types of inhomogeneous source terms in the K and FW-H equations. Later in this paper, for supersonic sources, we will treat other source types. We need to give the solution for the following four types of wave equations: 


$$
\begin{gathered}
\square^{2} p^{\prime}=\frac{\bar{\partial}^{2}}{\partial x_{i} \partial x_{j}}\left[T_{i j} H(f)\right], \\
\square^{2} p^{\prime}=Q_{1}(x, t) \delta(f), \\
\square^{2} p^{\prime}=\frac{\partial}{\partial t}\left[Q_{2} \delta(f)\right], \\
\square^{2} p^{\prime}=\nabla \cdot\left[Q_{3} \delta(f)\right] .
\end{gathered}
$$

This approach has several advantages. First the solution of each of these equations are basically different. Second, by seeking exact closed form solutions of these equations, one is able to evaluate various kinds of common approximations, e.g., mean surface approximation of blade loads, used in practical problems. It has been our policy at Langley to derive exact solutions to these equations suitable for development of robust and efficient noise prediction codes. We have always required that no approximations to the blade geometry and kinematics be made in the derivation of the main formulations. Various choices of approximations are made later starting from these results.

Because of the presence of the Dirac delta function in Eqs. (6-8), it is obvious that the sources on the right of these equations are surface sources. The nature of the source in Eq. (5) requires some explanation. From one point of view, it can be treated as a volume source while from another as a combination of volume and surface sources. We will elaborate further on this matter in the next section.

By the Kirchhoff method we mean any method based on the solutions of the $\mathrm{K}$ or FW-H equations. As seen above, the two equations are closely related in their derivation and solution. We will call the surface $f=0$ the data surface.

Remarks- 1. For many years, the FW-H equation was used in applications assuming that the data surface $f=0$ coincided with the blade surface, i.e., an impenetrable surface. Ffowcs Williams himself had suggested that a penetrable surface, as we have assumed here, may be more suitable for noise prediction [15] by including the significant part of the quadrupole sources inside 
the surface in calculations. Di Francescantonio in Italy [16] and independently Brentner and Farassat in the U.S. [17] implemented the suggestion of Ffowcs Williams. Di Francescantonio showed the equivalence of this method to that based on the Kirchhoff formula for a moving surface of Farassat and Myers [14] in the far field. Brentner and Farassat showed that the method based on the FW-H equation with penetrable data surface is superior to that based on the Kirchhoff formula in the near field while the computational efforts were comparable when the quadrupole sources outside the surface $f=0$ were neglected. This produced a flurry of activities by aeroacousticians around the world to convert their Kirchhoff codes based on the K equation to that based on the FW-H equation. In fact, there does not seem to be any incentive to use the Kirchhoff formula for moving surfaces in rotating blade noise prediction because it requires that the data surface be in the linear region where the CFD accuracy is in question. However, the Kirchhoff formula for a moving surface is of interest in other fields such as in the electromagnetic radiation problem [9].

2. Brentner and Farassat [17] have shown that the inhomogeneous source term on the right of the FW-H equation can be written as follows:

$$
Q_{K}+\frac{\bar{\partial}^{2}}{\partial x_{i} \partial x_{j}}\left[T_{i j} H(f)\right]+\ldots .-\frac{\partial}{\partial t}\left[\rho u_{n} \delta(f)\right]
$$

i.e., as the sum of the source terms on the right of the $\mathrm{K}$ equation, $Q_{K}$ and terms involving products of the first order parameters of the flow. The latter terms are negligible compared to the source on the right of the K equation in the linear region. This explains the difference in behavior of the solutions of the two equations in the near field.

3. The solution of the K and FW-H equations is fairly simple for subsonic surfaces. If part or all the surface $f=0$ travels at supersonic speed, obtaining a useful solution for computation is notoriously difficult. There are mathematical singularities in the solution that are not physical. There is cancellation of singularities among the solution of Eqs. (5-8). There is formation of shock-like structures and appearance of caustics in the acoustic field. We have spent much effort to derive solutions valid for supersonic surface noise prediction and to understand the structure of the radiation field. 
4. There seems to be endless varieties of solutions of the K and FW-H equations. One reason given in deriving a new solution appears to be that the available solutions are time consuming on a computer. In general, authors rarely state the computation time in research papers. It is so much a function of the algorithms used in the code and how experienced the researchers are in efficient code development. Moreover, computation time is computer dependent and computer technology is advancing at a very fast pace. At present there does not appear to be a need for deriving yet another solution to these equations for subsonic surfaces.

5. Because of the simplicity of the Green's function of the wave equation in the frame fixed to the undisturbed medium, it is better to always start in this frame for obtaining a solution even for the observer in motion. Generally, the known solutions in the moving frame are valid for uniform rectilinear motion and, thus, do not apply to an accelerating or maneuvering aircraft and somewhat restricted in the scope of application. This comment applies to open rotors and propellers. For ducted fan problems, often the frame of choice is one that is fixed to the duct.

\subsection{The Solution of the Governing Wave Equations}

We will now describe how Eqs. (5-8) are solved. We refer the readers to the appropriate papers for the details. It is not possible to give all the relevant details here because the algebraic manipulations are somewhat extensive.

\subsection{The Quadrupole Noise}

The quadrupole noise is governed by Eq. (5). This term only appears in the FW-H equation. Ideally, we would like to locate the data surface where the contribution of the quadrupoles outside the surface to the noise is small. At present in some important problems, such as in high speed helicopter rotor noise prediction, this does not seem possible because the accuracy of the CFD data is not satisfactory where the data surface must be positioned. The alternative technique is taking the blade surface as the data surface and adding the contribution of the quadrupoles in the regions where they have large source strength, to the surface noise. This requires a suitable solution of Eq. (5) as well as a good approximation method based on the physics of the problem. We will first present some exact results. 
Let $(\boldsymbol{x}, t)$ and $(\boldsymbol{y}, \tau)$ be the observer and the source space-time variables. For $r=|\boldsymbol{x}-\boldsymbol{y}|$, the surface $\Omega: r-c(t-\tau)=0$ defines a sphere with center at the observer and the radius $c(t-\tau)$ for all $\tau \leq t$. Here $c$ is the speed of sound in the undisturbed medium. Let $d \Omega$ be the element of the surface area of this sphere and define $F(\boldsymbol{y} ; \boldsymbol{x}, t)=[f(\boldsymbol{y}, \tau)]_{r e t} \equiv f(\boldsymbol{y}, t-r / c)$. Then the solution of Eq. (5) is

$$
4 \pi p^{\prime}(\boldsymbol{x}, t)=\frac{\partial^{2}}{\partial x_{i} \partial x_{j}} \int_{-\infty}^{t} \frac{d \tau}{t-\tau} \int_{\Omega} H(F)\left[T_{i j}\right]_{r e t} d \Omega(\boldsymbol{y})
$$

One can visualize the above integral dynamically. In what follows, we assume that $(\boldsymbol{x}, t)$ is kept fixed in the discussion. The surface $\Omega$ is called the collapsing sphere because, this surface represents a sphere with center at $\boldsymbol{x}$ whose radius collapses at speed of sound. Figure 1 shows the collapsing sphere in the process of intersecting a rotor blade. The surface $F=0$ is generated by the intersection of the collapsing sphere and the data surface $f=0$ for $\tau \leq t$. This surface can be determined for a fixed $(x, t)$ in advance and is independent of the source time. The inner surface integral in Eq. (9) sums the contribution of all the quadrupoles outside the surface $F=0$ over the sphere $\Omega$ at the source time $\tau$. As $\tau$ varies, the radius of $\Omega$ changes and therefore this surface integral is source time dependent. The result of the surface integral is, therefore, a function of $(\tau ; \boldsymbol{x}, t)$. The outer integral sums this function over the source time with the denominator of the integrand accounting for the spherical spreading of the radiated sound.

The above integral treats all the quadrupoles as volume sources. It is an exact result and is valid even when shock waves, wakes and other discontinuities are present in the flow. In practical problems, the integral on the right side of Eq. (9) is evaluated numerically and one is therefore obliged to use numerical differentiation with respect to the observer variables- a procedure that is subject to numerical errors in addition to being time consuming on a computer. Farassat and Brentner have converted the above double differentiation with respect to the space variables to derivatives with respect to observer time variable [18]. The conversion can be performed exactly. The procedure is similar to derivation of Formulation 1 in Subsection 3.3.1. This result was used by Brentner and Holland [19] and Farassat and Brentner [20] to derive 
two analytic approximations to Eq. (9) called Formulations Q1A and Q2, respectively, for prediction of high speed impulsive noise of helicopter rotors.

Both these formulations approximate the surface $\Omega$ by a portion of a cylinder with axis passing through the observer and perpendicular to rotor plane. The observer is assumed to be in the far field and in the rotor plane where the peak directivity of high speed impulsive noise lies. The quadrupole sources are integrated along lines perpendicular to the rotor plane and treated as new surface sources on this plane. Although this approximation is strictly valid for the observer in the far field and in the rotor plane, tests have shown that it is also good for the observer out of the rotor plane. Two different analytic techniques were used to get the two formulations. Formulation Q1A is valid for subsonic sources because of the appearance of the Doppler factor in the denominator of its integrand. Formulation Q2 is valid for both subsonic and supersonic sources and is remarkably simple. The use of the above approximation yields a substantial reduction in quadrupole source strength storage space and computation time. In fact, it can be said that quadrupole noise prediction has now come within the reach of helicopter rotor designers.

We summarize the most important conclusions of the research on high speed rotor noise prediction at Langley from the numerical studies based on the above two formulations $[19,20]$ :

1. Formulation Q1A produces a more robust code than Formulation Q2 because the former has time derivatives while the latter has directional derivatives of the surface sources. Because of high source gradients at the leading edge of the blades and across shock traces, Formulation Q2 is prone to numerical errors.

2. Shock surfaces and the region near the leading edge of the blades contribute significantly to the high speed rotor noise. We explain in the next Subsection how shock surfaces that have no volume can actually contribute to the quadrupole noise.

3. In case of delocalization [21] where the shocks on the blades can extend far beyond the blade tips, one should also include the source region beyond the tips in noise prediction. However, the sources over the blades and beyond the tips behave differently in shaping the acoustic waveform. The sources around and in the vicinity of the blades account for the peak level of the 
waveform but the sources beyond the tip account for the steepening and broadening of the waveform which can often be significant.

\subsection{Is the Quadrupole Source Term in Eq. (5) a Volume Source?}

The inhomogeneous source term of Eq. (5) bears much resemblance to that in Lighthill's jet noise equation. If we study the derivation of FW-H equation carefully, we recognize that we have explicitly exhibited only the discontinuity of fluid parameters across the surface $f=0$. There may be other discontinuities within the fluid such as shock surfaces, thin wakes and vortices. We can explicitly exhibit the sources due to these discontinuities by using just the concept of generalized differentiation $[6,7,8,12,13]$. Farassat, Brentner and Myers [18, 22, 23] have carried out this procedure in detail. To illustrate the method we will only consider the discontinuity across a shock wave in the flow and show how easily we can identify the sources on the shocks and their strengths.

Let the shock surface be defined again by $f=0$. We consider the quadrupole source term of Lighthill

$$
Q=\frac{\bar{\partial}^{2} T_{i j}}{\partial x_{i} \partial x_{j}}
$$

The derivatives here are generalized derivatives as indicated in this equation by a bar over the derivative sign. Understanding and acceptance of this statement requires much thinking and mathematical maturity. It follows from the fact that conservation laws and all the steps in deriving the FW-H equation are set in the space of generalized functions. Thus discontinuous functions are naturally allowed in the solution of differential equations but all derivatives are generalized derivatives. To avoid confusion with the notation of ordinary derivatives, we use a bar over the derivative sign whenever there is the danger of confusion. We have eliminated the Heaviside function of the source term of Eq. (5) to illustrate the main idea and to simplify algebraic manipulations. The rule of generalized differentiation gives us in two steps the following: 


$$
\begin{aligned}
& Q=\overline{\frac{\partial}{\partial x_{i}}}\left[\frac{\partial T_{i j}}{\partial x_{j}}+\Delta T_{i j} n_{j} \delta(f)\right] \\
& =\frac{\partial^{2} T_{i j}}{\partial x_{i} \partial x_{j}}+\Delta\left(\frac{\partial T_{i j}}{\partial x_{j}}\right) n_{i} \delta(f) \\
& +\frac{\partial}{\partial x_{i}}\left[\Delta T_{i j} n_{j} \delta(f)\right]
\end{aligned}
$$

where $\Delta=[.]_{2}-[.]_{1}$ stands for the jump of a quantity across the shock, the subscripts 1 and 2 refer to the two sides of the shock surface and $n_{i}$ denotes the components of the local unit normal to this surface pointing into region 2. It is now obvious that the jump across a shock produce sources of the types in Eqs. (6) and (8). They can be recognized as a monopole and a dipole distribution on the shock surface. All the derivatives, except in the last term on the right of Eq. (11) are ordinary derivatives now. Using the mass continuity and momentum equations, one can write the jumps in Lighthill stress tensor and its derivatives across the shock in terms of other flow parameters that are computed in CFD codes.

We discuss a subtle point about Lighthill's jet noise theory when shocks are present in the flow. It can be shown that the derivatives of the inhomogeneous source term of Lighthill's equation must be treated as generalized derivatives in this case, i.e., the source term is precisely $Q$ in Eq. (10) [5]. Equation (11) tells us that when there are shocks inside a jet, then $Q \neq \partial^{2} T_{i j} / \partial x_{i} \partial x_{j}$. Note that the term $\partial^{2} T_{i j} / \partial x_{i} \partial x_{j}$ in Eq. (11) has ordinary derivatives. This term has a discontinuity across the shock and thus some of the algebraic manipulations of this term under an integral are different from those associated with shock free jet noise theory. For example, the following two integrals are not equal because of this discontinuity:

$$
I_{1}=\int \frac{1}{r}\left[\frac{\partial^{2} T_{i j}}{\partial y_{i} \partial y_{j}}\right]_{r e t} d \boldsymbol{y}
$$




$$
I_{2}=\frac{\partial^{2}}{\partial x_{i} \partial x_{j}} \int \frac{\left[T_{i j}\right]_{r e t}}{r} d \boldsymbol{y}=\int \frac{1}{r}\left[\frac{\bar{\partial}^{2} T_{i j}}{\partial y_{i} \partial y_{j}}\right]_{r e t} d \boldsymbol{y}
$$

It is clear that $I_{1}$ is the contribution of $\partial^{2} T_{i j} / \partial x_{i} \partial x_{j}$ to $4 \pi p^{\prime}(x, t)$ while $I_{2}$ is the full contribution of $Q$. This means that $p^{\prime}(x, t)=I_{2} / 4 \pi$ is the solution of the jet noise equation when shocks are present in the flow. Furthermore, if we use Eq. (11) in the integrand of the last integral on the right side of Eq. (13), we will get $I_{1}$ plus some surface integrals over the shock surfaces inside the jet. The subtlety we refer to here is that numerically this result is equal to that obtained from the expression on the right of the first equality sign in Eq. (13). But this expression happens to be the familiar solution that Lighthill gave for his shock free jet noise theory.

Farassat and coworkers had proposed the rotating shock waves on rotor blades as the main source of high speed impulsive noise [24]. Some shock noise calculations based on Eq. (11) have shown this to be basically correct. It was found later using Formulation Q2 that the quadrupole sources near the leading edge of the blades is another important contributor to high speed rotor noise [20]. Currently the main obstacle in utilizing Eq. (11) for shock noise prediction is that one requires high resolution CFD data to get the source strengths. At present, CFD codes can not provide the jump in the gradient of the Lighthill stress tensor across the shock accurately in reasonable computation time for noise prediction. One should note that CFD code developers for aerodynamic calculations are generally not interested in this parameter. They should be made aware of such a need in aeroacoustics.

Many interesting results await discovery by studying the quadrupole source term of FW-H equation in the setting of generalized function theory. In addition to shock sources, other sources from wake flapping, vortex motion and boundary layer can easily be identified [22, 23]. Unfortunately, because of the heavy investment in time required to learn the needed mathematical tools which include differential geometry and general tensor analysis, researchers have not been attracted to the study of these sources by this method. It is to be noted that intuitively, one may not recognize rotating shocks and flapping wakes as sources of sound. And if one does so, finding the source strengths from classical analysis is not an easy job because of substantial entanglement 
in physical arguments and algebraic manipulations. The method proposed here is the natural approach because discontinuities are allowed in generalized functions and all the operations on ordinary functions are at our disposal with remarkable properties such as exchange of limit operations.

So what is the answer to the question in the title of this Subsection? The quadrupole source in Eq. (5) is a volume source only if the solution is written in the form of Eq. (9) or its equivalents such as Formulations Q1A and Q2. The integrand in this equation can and usually is discontinuous due to shocks, wakes, etc. We can break down the quadrupole source term of Eq. (5) separating the contributions of the discontinuities which appear as surface terms involving jumps in Lighthill stress tensor and its gradient across the surface of discontinuity. So the source term is Eq. (5) can also be viewed as a combination of volume and surface sources the sum of whose contributions to the noise is the same as that from Eq. (9).

\subsection{The Surface Noise - Equations (6-8)}

We start by giving a solution of Eq. (6) that is both simple and basic for the wave equation with sources on a moving surface $[12,13,25]$ :

$$
4 \pi p^{\prime}(\boldsymbol{x}, t)=\int_{F=0} \frac{1}{r}\left[\frac{Q_{1}(\boldsymbol{y}, \tau)}{\Lambda}\right]_{r e t} d \Sigma
$$

where the parameter $\Lambda$ is

$$
\Lambda=\left(1+M_{n}^{2}-2 M_{n} \cos \theta\right)^{1 / 2}
$$

Here, $M_{n}$ is the local normal Mach number of the surface $f=0$ and $\theta$ is the angle between $\boldsymbol{n}$ and the radiation direction $\hat{\boldsymbol{r}}=(\boldsymbol{x}-\boldsymbol{y}) / r$. The surface $F=0$ defined earlier is called the influence surface of $(\boldsymbol{x}, t)$ or more commonly the $\Sigma$-surface. Its construction is shown in Figs. 1 and 2. More details can be found in [25]. The following relations derived by Farassat [26], can be used to write the above solution in two other forms: 


$$
\frac{d \Sigma}{\Lambda}=\frac{d S}{\left|1-M_{r}\right|}=\frac{c d \Gamma d \tau}{\sin \theta}
$$

where $d S$ is the element of surface area of the data surface $f=0, M_{r}$ is the local Mach number in the radiation direction of the data surface and $d \Gamma$ is the element of length of the curve of intersection of the collapsing sphere and the the data surface (See Fig. 1).

\subsubsection{The Subsonic Case}

The solution of Eq. (7) for a data surface that moves subsonically is based on Eqs. (15) and (16). It is

$$
4 \pi p^{\prime}(\boldsymbol{x}, t)=\frac{\partial}{\partial t} \int_{f=0}\left[\frac{Q_{2}(\boldsymbol{y}, \tau)}{r\left|1-M_{r}\right|}\right]_{r e t} d S
$$

To bring in the time derivative, we use the following relation

$$
\frac{\partial[q(\boldsymbol{y}, \tau)]_{r e t}}{\partial t}=\left[\frac{1}{1-M_{r}} \frac{\partial q(\boldsymbol{y}, \tau)}{\partial \tau}\right]_{r e t}
$$

where $q(\boldsymbol{y}, \tau)$ is the expression inside the square brackets in the integrand of Eq. (17). We will not carry out this step.

The solution of Eq. (8) is also based on Eqs.(15) and (16) and is:

$$
4 \pi p^{\prime}(\boldsymbol{x}, t)=\nabla \cdot \int_{f=0}\left[\frac{\boldsymbol{Q}_{3}(\boldsymbol{y}, \tau)}{r\left|1-M_{r}\right|}\right]_{r e t} d S
$$

To bring the space derivative in, we prefer to convert it first to time derivative and then use Eq. (18). This conversion was given by Farassat [26, 27] based on an identity relating the space and time derivatives of the Green's function of the Wave equation. The result is: 


$$
\begin{gathered}
4 \pi p^{\prime}(\boldsymbol{x}, t)=-\frac{1}{\bar{c}} \frac{\partial}{\partial t} \int_{f=0}\left[\frac{Q_{3 r}(\boldsymbol{y}, \tau)}{r\left|1-M_{r}\right|}\right]_{r e t} d S \\
-\int_{f=0}\left[\frac{Q_{3 r}(\boldsymbol{y}, \tau)}{r^{2}\left|1-M_{r}\right|}\right]_{r e t} d S
\end{gathered}
$$

where we have defined $Q_{3 r}=\boldsymbol{Q}_{3} \cdot \hat{\boldsymbol{r}}$. When $Q_{2}=\rho_{0} v_{n}$ and $\boldsymbol{Q}_{3}=-p \boldsymbol{n}$, Eqs. (17) and (20) give the thickness and loading noise terms of Formulation 1 of Farassat, respectively [26-28]. The data surface $f=0$ is taken as the blade surface itself. After taking the time derivative inside the integrals by using Eq. (18), we get the thickness and loading noise terms of Formulation 1 A of Farassat [28, 29]. This formulation has been coded in NASA helicopter noise prediction code WOPWOP [29] which is extensively used by the U.S. helicopter industry. This code is highly robust and efficient on a desktop workstation. Some versions of the code also have the capability of predicting the subsonic and supersonic quadrupole noise. There are many publications by NASA and industry researchers and engineers reporting on the application of this code to helicopter rotor noise prediction and comparison with experimental data.

\subsubsection{The Supersonic Case}

For supersonically moving surfaces it is difficult to get a singularity free solution of the FW-H equation which can be used in the development of an efficient and robust code. In general, the formulations are very complicated and their derivation requires many algebraic manipulations [30, 31]. In noise prediction codes, the data surface is divided into panels and the contribution of each panel to the noise is evaluated separately and summed. Naturally, for panels moving at subsonic speed, we want to use the simple subsonic formulations such as $1 \mathrm{~A}$ discussed above. It can be shown that no additional terms appear in this formulation, e.g., edge terms, when we use it for a panel. For supersonic formulations, in general, one has edge line integrals in the solution of the wave equation for a panel. Therefore, we should explicitly write the surface source terms of FW-H or K equations for a panel as follows. Let $\tilde{f}(x, t)=0$ be defined such that $f=\tilde{f}=0$ describes the edge of the panel 
and $\nabla \tilde{f}=v$, where $v$ is the unit inward geodesic normal at the edge. This latter condition implies that $\tilde{f}>0$ on the panel. The geodesic normal is a vector that is tangent to the panel and simultaneously perpendicular to the edge curve. Let $H(\tilde{f})$ denote the Heaviside function. We are interested in solving the following three wave equations when the panel described by $f=0, \tilde{f}>0$ moves at arbitrary supersonic speed:

$$
\begin{aligned}
\square^{2} p^{\prime} & =Q_{1}(\boldsymbol{x}, t) H(\tilde{f}) \delta(f) \\
\square^{2} p^{\prime} & =\frac{\partial}{\partial t}\left[Q_{2} H(\tilde{f}) \delta(f)\right] \\
\square^{2} p^{\prime} & =\nabla \cdot\left[Q_{3} n H(\tilde{f}) \delta(f)\right]
\end{aligned}
$$

The solution of Eq. (21) is just slightly different from that of Eq. (6):

$$
4 \pi p^{\prime}(\boldsymbol{x}, t)=\int_{\substack{F=0 \\ \tilde{F}>0}} \bar{r}\left[\frac{Q_{1}(\boldsymbol{y}, \tau)}{\Lambda}\right]_{r e t} d \Sigma
$$

where $\tilde{F}(\boldsymbol{y} ; \boldsymbol{x}, t)=[\tilde{f}(\boldsymbol{y}, \tau)]_{\text {ret }}$. The solutions of Eqs. (22) and (23) are more complicated. We take the following step to reduce algebraic manipulation $[12,13]$. Any function multiplying the Dirac delta function can be restricted to the support of the delta function. For example, it is well-known that $\varphi(x) \delta(x)=\varphi(0) \delta(x)$. Using a tilde under a symbol to denote restriction of a function to the panel surface and the rules of differentiation in generalized function theory[6-8, 12, 13], we get 


$$
\begin{aligned}
& \frac{\partial}{\partial t}\left[Q_{2} H(\tilde{f}) \delta(f)\right]=\frac{\partial}{\partial t}\left[{\underset{\sim}{2}}_{2} H(\tilde{f}) \delta(f)\right] \\
& =\frac{\partial \underline{\sim}_{2}}{\partial t} H(\tilde{f}) \delta(f)-c M_{v}{\underset{\sim}{2}}_{2} \delta(\tilde{f}) \delta(f) \\
& -c M_{n} Q_{2} H(\tilde{f})|\nabla f| \delta^{\prime}(f) \\
& \begin{aligned}
\nabla \cdot\left[\boldsymbol{Q}_{3} H(\tilde{f}) \delta(f)\right]= & \nabla \cdot\left[{\left.\underset{\sim}{\boldsymbol{Q}_{3}} H(\tilde{f}) \delta(f)\right]}_{=}\right. \\
& \left(\nabla_{2} \cdot{\left.\underset{\sim}{\boldsymbol{Q}_{3 T}}-2 H_{f}{\underset{\sim}{3 n}}_{3 n}\right) H(\tilde{f}) \delta(f)}+{\underset{\sim}{3 v}}_{3 v} \delta(\tilde{f}) \delta(f)+{\underset{\sim}{3 n}}_{3 n} H(\tilde{f})|\nabla f| \delta^{\prime}(f)\right.
\end{aligned}
\end{aligned}
$$

Here, we have the following symbols: $\underset{\sim}{Q_{3 v}}=\underset{\sim}{\boldsymbol{Q}_{3}} \cdot v, M_{v}=\boldsymbol{M} \cdot v$ is the local Mach number in the direction of the geodesic normal, ${\underset{\sim}{3 T}}_{3 T}$ is the component of $\underset{\sim}{\boldsymbol{Q}_{3}}$ tangent to the panel, $\nabla_{2} \cdot \boldsymbol{Q}_{3 T}$ is the surface divergence of ${\underset{\sim}{\boldsymbol{Q}}}_{3}$, ${\underset{\sim}{3 n}}_{3 n}$ is the component of $\underset{\sim}{Q_{3}}$ normal to the panel and $H_{f}$ is the local mean curvature of the panel surface. Strictly speaking, we do not need the restriction sign over many of the symbols. However, the sign is necessary on $\partial{\underset{\sim}{Q}}_{2} / \partial t$, and on the symbols multiplying $\delta^{\prime}(f)$ as shown. Note that $M_{n}$ multiplying $\delta^{\prime}(f)$ in Eq. (25), and $|\nabla f|$ in both of the above equations multiplying $\delta^{\prime}(f)$ are not restricted to the surface of the panel [32]. Therefore, we can not set $|\nabla f|=1$ in these terms.

We can always set $\partial \underset{\sim}{q} / \partial n=0$ for any function $q(\boldsymbol{x}, t)$ which is restricted to the surface $f=0$. This property helps in the reduction of algebraic manipulations which are extensive in the derivation of a supersonic formulation. The time derivative of the restricted function is given by the relation $\partial \underset{\sim}{q} / \partial t=\partial q / \partial t-v_{n} \partial q / \partial n$ which is what an observer sitting on the data surface measures as the rate of change of $q$ with time. In this relation $v_{n}$ is the local normal velocity of the data surface. To illustrate the nature of the 
simplification introduced by restriction of a function multiplying Dirac delta function to the support of the delta function, we give the following example. Taking the derivatives with respect to $x$ of both sides of the equation $\varphi(x) \delta(x)=\varphi(0) \delta(x)$, we get $\varphi^{\prime}(x) \delta(x)+\varphi(x) \delta^{\prime}(x)=\varphi(0) \delta^{\prime}(x)$. This relation means that the functional values of the generalized functions on each side of the equality sign are the same for any test function. Obviously the generalized function on the right side of this relation is simpler than that on the left side. In the multi-dimensional case of our problem, the simplification in algebraic manipulations is substantial because the number of expressions involved in the derivation is reduced.

The first source terms of Eqs. (25) and (26) is of the type in Eq. (21). We need to give the solution to two new wave equations with sources of the types below:

$$
\begin{gathered}
\square^{2} p^{\prime}=Q_{4} \delta(\tilde{f}) \delta(f) \\
\square^{2} p^{\prime}=Q_{5} H(\tilde{f})|\nabla f| \delta^{\prime}(f)
\end{gathered}
$$

The solution of Eq. (27) is given in [12] as follows:

$$
4 \pi p^{\prime}(\boldsymbol{x}, t)=\int_{\substack{F \\ \tilde{F}=0}} \bar{r}\left[\frac{Q_{4}}{\Lambda_{0}}\right]_{r e t} d L
$$

where $\Lambda_{0}=|\nabla F \times \nabla \tilde{F}|$ which can be written in terms of the geometric and kinematic parameters of the panel[12], and $\mathrm{dL}$ is the element of the length of the edge of the $\Sigma$-surface generated by the panel surface.

The solution of Eq.(28) is much harder to obtain. After using the Green's function of the wave equation and integrating with respect to the source time, we get

$$
4 \pi p^{\prime}(\boldsymbol{x}, t)=\int \frac{1}{r}\left[Q_{5}|\nabla f|\right]_{r e t} H(\tilde{F}) \delta^{\prime}(F) d \boldsymbol{y}
$$

We next use the following identity [12, Eq. (4.39), 32] to interpret the above integral: 


$$
\int \phi(x) \delta^{\prime}(f) d y=\int_{f=0}\left\{-\frac{1}{|\nabla f|} \frac{\partial}{\partial n}\left[\frac{\phi(x)}{|\nabla f|}\right]+\frac{2 H_{f} \phi(x)}{|\nabla f|^{2}}\right\} d S
$$

where $f$ is an arbitrary surface here which is replaced by $F$ when this identity is applied to Eq. (30). Here $H_{f}$ denotes the local mean curvature of the surface $f$. For the $\Sigma$-surface of the panel, let $N=\left(\boldsymbol{n}-M_{n} \hat{\boldsymbol{r}}\right) / \Lambda$ be the local unit normal and $H_{F}$ be the local normal curvature. It can be shown easily that $|\nabla F|=\Lambda[|\nabla f|]_{\text {ret }}$. Equation (30) can then be written as:

$$
4 \pi p^{\prime}(x, t)=\int_{F=0}\left\{-\frac{1}{\Lambda} \frac{\partial}{\partial N}\left[\frac{\left[Q_{5}\right]_{r e t} H(\tilde{F})}{r \Lambda}\right]+\frac{2 H_{F}\left[Q_{5}\right]_{r e t} H(\tilde{F})}{r \Lambda^{2}}\right\} d \Sigma(\mathrm{EQ} \mathrm{32)}
$$

Our task in finding a solution of Eq. (28) is not finished. The first term in the integrand gives a line integral because we have

$$
\frac{\partial H(\tilde{F})}{\partial N}=N \cdot \nabla \tilde{F} \delta(\tilde{F})
$$

The interpretation of the resulting integral is [12]:

$$
\int_{F=0} \frac{\left[Q_{5}\right]_{r e t} N \cdot \nabla \tilde{F} \delta(\tilde{F})}{r \Lambda^{2}} d \Sigma=\int_{\substack{F=0 \\ \tilde{F}=0}} \frac{\left[Q_{5}\right]_{r e t} \cot \theta^{\prime}}{r \Lambda^{2}} d L
$$

where $\theta^{\prime}$ is the angle between $\nabla F$ and $\nabla \tilde{F}$. See references [12, 13] for further details of the above mathematical steps.

We have, thus, given the full solution of the FW-H and the K equations for sources on a panel moving at any speed but, in particular at supersonic speed. We believe that this result is suitable for development of an efficient computer code so long as we limit the use to supersonic panels [31]. For the thickness and loading terms of the FW-H equation, the resulting analytic 
expression after taking all the derivatives explicitly, is a very complicated result known as the Formulation 3 of Farassat [30, 31]. Formulation 3 has been coded in the high speed propeller noise prediction code ASSPIN [33] by Mark H. Dunn. The derivation here is different from and more advanced than the original derivation in the early Eighties. Farassat and Farris [25] have recently published the full derivation of the mean curvature of the $\Sigma$ - surface which was originally derived and published in 1996 by the first author [13].

In propfan noise prediction, the line integral in Formulation 3 can produce a logarithmic singularity in the acoustic field when the leading and trailing edges are not sufficiently swept and the propeller is operated at high supersonic tip speed. This singularity was studied by E. De Bernardis in a Ph.D. dissertation of University of Rome (La Sapienza) and reported later in [34]. Farassat and Myers [35] have shown that the singularity is removed if we add the contribution of the quadrupoles of the FW-H equation to the line integral. Thus, the singularity of the line integral in Formulation 3 is because the physics of the problem is not accounted fully in the differential equation when we retain only the thickness and loading source terms of the FW-H equation.

We have continued to work on the supersonic source problem. A more recent paper from a totally different direction is reported by Farassat, Brentner and Dunn [36]. One interesting result of this work is that the analytic expression for the acoustic pressure obtained, called Formulation 4, is closely related to those of geometrical acoustics and optics. Another interesting and important result is the formation of caustics in the radiation field, in particular in the near field, of rotating blades at all tip speed range. In fact, it appears that for further progress, we should apply other areas of advanced mathematics such as the singularity theory [37] as utilized, for example, in optics [38]. Some interesting work along this line is by Ardavan [39, 40].

In this paper, we have emphasized the work of the author and coworkers at NASA Langley Research Center. The readers are encouraged to study the publications of the following researchers who have contributed to time domain analysis and its applications in rotating blade noise prediction: M. K. Myers, Kenneth S. Brentner, T. F. Brooks and C. L. Burley (blade-vortex interaction noise, tilt rotor noise), D. L. Hawkings and M. V. Lowson, H. Ardavan, H. Tadghighi, Lyle N. Long, A. R. George (rotor noise), A. S. Lyrinzis (application of Kirchhoff formulas to rotor noise problems, particu- 
larly the review article [41]), Michael Carley (propeller noise), Martin Kuntz (rotor noise), Judith M. Gallman (application of Formulation 1 to rotor noise), S. Ianniello (rotor noise, particularly the papers on the construction of the $\Sigma$-surface), Luigi Morino (BEM in aerodynamics and aeroacoustics), P. Di Francescantonio (rotor noise) and Enrico De Bernardis, V. Wells and A. Han (rotor noise).

\subsection{Concluding Remarks}

We have reviewed the advances in the application of the Kirchhoff method in discrete frequency noise prediction. Much progress has been made in the past thirty years. It can be said that for aeroacoustic applications one can depend exclusively on the FW-H equation with a penetrable data surface enclosing the most significant part of the quadrupole sources. It has been shown that the data surface can even be in the nonlinear region where the use of the Kirchhoff formula for a moving surface, a solution of the K equation, would give large errors [17]. The state of noise prediction for subsonic sources is highly satisfactory. We have a solution of the FW-H equation that has given us an efficient and robust code. When part or all of the data surface $f=0$ moves at supersonic speed, both the structure of the acoustic field and the mathematics become much more complicated. Part of the mathematical problem is due to the appearance of singularities in the analytic results which are not real. There is also cancellation of singularities among various integrals. Much progress has been made in the supersonic case. We have learned a lot but there is more to explore about this problem in the future. It was my encounter with Phil Doak in 1973 that set the direction of my research in time domain analysis. His farsighted remark about ignoring "the force of tradition" made the time domain calculations routine these days for helicopter rotor and propeller noise prediction. Happy eightieth birthday Phil!

\subsection{References}

1. L. Gutin 1948 NACA TM 1195. On The sound field of a rotating propeller, (Original in Russian in 1936 Zhurnal Tekhnicheskoi Fiziki 12, 76-83)

2. M. V. Lowson 1965 Proc. Roy. Soc. London A286, 559-572. The sound field for singularities in motion 
3. W. Ernsthausen 1936 Luftfahrtforschung 13(12). The source of propeller noise. Translated as NACA TM 825, 1937

4. A. F. Deming 1937, 1938 NACA TN $605 \& 679$. Noise from propellers with symmetrical sections at zero blade angle, I \& II

5. J. E. Ffowcs Williams and D. L. Hawkings 1969 Phil. Trans. Roy. Soc.

London A264, 321-342. Sound generation by turbulence and surfaces in arbitrary motion

6. I. M. Gelfand and G. E. Shilov 1964 Generalized Functions. 1- Properties and Operations, Academic Press

7. D. S. Jones 1982 The Theory of Generalized Functions. Second Edition, Cambridge University Press

8. Ram P. Kanwal 1998 Generalized Functions- Theory and Technique, second edition, Birkhauser, Boston

9. D. S. Jones 1964 The Theory of Electromagnetism. Pergamon Press

10. N. Curle 1955 Proc. Roy. Soc. London A231, 505-514. The influence of solid boundaries on aerodynamic sound

11. M. E. Goldstein 1976 Aeroacoustics. New York, McGraw-Hill

12. F. Farassat 1994 NASA Technical Paper 3428 Introduction to generalized functions with applications in aerodynamics and aeroacoustics, Corrected Copy, April 1996 (obtainable from ftp://techreports.larc.nasa.gov/pub/techreports/larc/94/tp3428.ps.Z)

13. F. Farassat 1996 NASA Technical Memorandum 110285. The Kirchhoff formulas for moving surfaces in aeroacoustics- The subsonic and supersonic cases (obtainable from ftp://techreports.larc.nasa.gov/pub/techreports/larc/ 96/NASA-96-tm110285.ps.Z)

14. F. Farassat and M. K. Myers 1988 J. Sound and Vib. 123, 451-560. Extension of Kirchhoff's formula to radiation from moving surfaces 
15. D.G. Crighton, A. P. Dowling, J. E. Ffowcs Williams, M. Heckl, and F. G. Leppington 1992 Modern Methods in Analytical Acoustics- Lecture Notes, Springer-Verlag

16. P. Di Francescantonio 1997 Jour. Sound and Vib. 202(1), 491-509. A new boundary integral formulation for prediction of sound radiation

17. Kenneth S. Brentner and F. Farassat 1998 AIAA Journal 36(8),1379-1386. An analytical comparison of the acoustic analogy and kirchhoff formulation for moving surfaces. (obtainable from http://techreports.larc.nasa.gov/ltrs/ PDF/1998/jp/NASA-98-aj-ksb.pdf)

18. F. Farassat and Kenneth S. Brentner 1988 AHS J. 33(1), 29-36. The uses and abuses of the acoustic analogy in helicopter rotor noise prediction

19. Kenneth S. Brentner and Peter C. Holland 1997 AHS J. 42(2), 172-181. An Efficient and Robust Method for Computing Quadrupole Noise

20. F. Farassat and Kenneth S. Brentner 1998 Jour. Sound and Vib. 218(3), 481-500. Supersonic quadrupole noise theory for high-speed helicopter rotors

21. Y. H. Yu, F. X. Caradonna and F. H. Schmitz 1978 Fourth European Rotorcraft and Power Lift Aircraft Forum, Paper 58. The influence of the transonic flow field on high-speed helicopter impulsive noise

22. F. Farassat 1987 AIAA-87-2675. Quadrupole source in prediction of the noise of rotating blades- A new source description

23. F. Farassat and M.K.Myers 1990 Computational Acoustics, Volume 2, Scattering, Gaussian Beams and Aeroacoustics, D. Lee, A. Cakmak, and R. Vichnevetsky (Eds.), Amsterdam, North Holland, 227-240. An analysis of the quadrupole noise source of high speed rotating blades

24. F. Farassat, Y.-J. Lee, H. Tadghighi and R. Holtz 1991. AHS/RAS International Technical Specialists' Meeting on Rotorcraft Acoustics and Rotor Fluid Dynamics. High-speed helicopter rotor noise- Shock waves as a potent source of sound 
25. F. Farassat and Mark Farris 1999 Math. Meth. Appl. Sci. 22, 1485-1503. The mean curvature of the influence surface of wave equation with sources on a moving surface

26. F. Farassat 1975, NASA Tech. Rep. R-451. Theory of noise generation from moving bodies with an application to helicopter rotors (Available at http://techreports.larc.nasa.gov/ltrs/PDF/NASA-75-trr451.pdf)

27. F. Farassat 1981 AIAA J. 19(9), 1122-1130. Linear acoustic formulas for calculation of rotating blade noise

28. F. Farassat and G. P Succi 1983 Vertica 7, 309-320. The prediction of helicopter rotor discrete frequency noise

29. Kenneth S. Brentner 1986 NASA Technical Memorandum 87721. Prediction of Helicopter rotor discrete frequency noise- A computer program incorporating realistic blade motions and advanced acoustic formulation

30. F. Farassat $1985 A G A R D-C P-366$. Theoretical analysis of linearized acoustics and aerodynamics of advanced supersonic propellers

31. F. Farassat, S. L. Padula, and M. H. Dunn 1987 Jour. of Sound and Vib., 119(1), 53-79. Advanced turboprop noise prediction based on recent theoretical results

32. F. Farassat 2000 Jour. of Sound and Vib., 230(2), 460-462. The integration of $\delta^{\prime}(f)$ in a multi-dimensional space

33. M. H. Dunn and G. M. Tarkenton 1992 NASA CR 4434. Computational methods in the prediction of advanced subsonic and supersonic propeller induced noise- ASSPIN User Manual

34. E. De Bernardis and F. Farassat 1990 Boundary Element Methods in Engineering, B. S. Annigeri and K. Tseng (Eds.), Springer-Verlag, 522-528, On the possibility of singularities in the acoustic field of supersonic sources when BEM is applied to a wave equation

35. F. Farassat and M. K. Myers 1994 Structural Acoustics, Scattering and Propagation, International Conference on Theoretical and Computational Acoustics, Volume 1, J. E. Ffowcs Williams, D. Lee and A. D. Pierce (Eds.), 
World Scientific, Singapore, 29-43.Line source singularity in the wave equation and its removal by quadrupole source- a supersonic propeller noise problem

36. F. Farassat, Kenneth S. Brentner and M. H. Dunn AIAA 98-2375, 1998. A study of supersonic surface sources-The Ffowcs Williams-Hawkings equation and the Kirchhoff formula, (corrected copy obtainable from ftp://techreports.larc.nasa.gov/pub/techreports/larc/1998/aiaa/NASA-aiaa-98-2375.ps.Z)

37. J. W. Bruce and P. J. Giblin 1992 Curves and Singularities, Second Edition, Cambridge University Press

38. J. F. Nye 1999 Natural Focussing and Fine Structure of Light, Bristol, Institute of Physics Publishing

39. H. Ardavan 1991 Proc. R. Soc. Lond. A433, 451-459. The near-field singularity predicted by the spiral Green's function in acoustics and electrodynamics.

40. H. Ardavan 1991 J. Fluid Mech. 226, 591-624. The breakdown of the linearized theory and the role of quadrupole sources in transonic rotor acoustics.

41. Lyrintzis, A. S.1995 ASME Journal of Fluids Engineering, 116, 665-676. Review: The use of Kirchhoff's method in computational aeroacoustics 


\section{Captions for Figures}

Figure 1. The collapsing sphere intersecting a rotating blade at the source time $\tau$. Note that the collapsing sphere is the intersection of the characteristic cone (conoid) of $(\boldsymbol{x}, t)$ with the three dimensional space at the time $\tau$.

Figure 2. The construction of the $\Sigma$-surface for a flat rectangular rotor blade. The rotor is moving forward at the velocity $V_{F}$ in such a way that all points on the rotor travel at subsonic speed relative to the speed of sound in undisturbed medium. The $\Sigma$ - surface will be more complicated and even in several pieces if part or all the blade surface travel at supersonic speed 


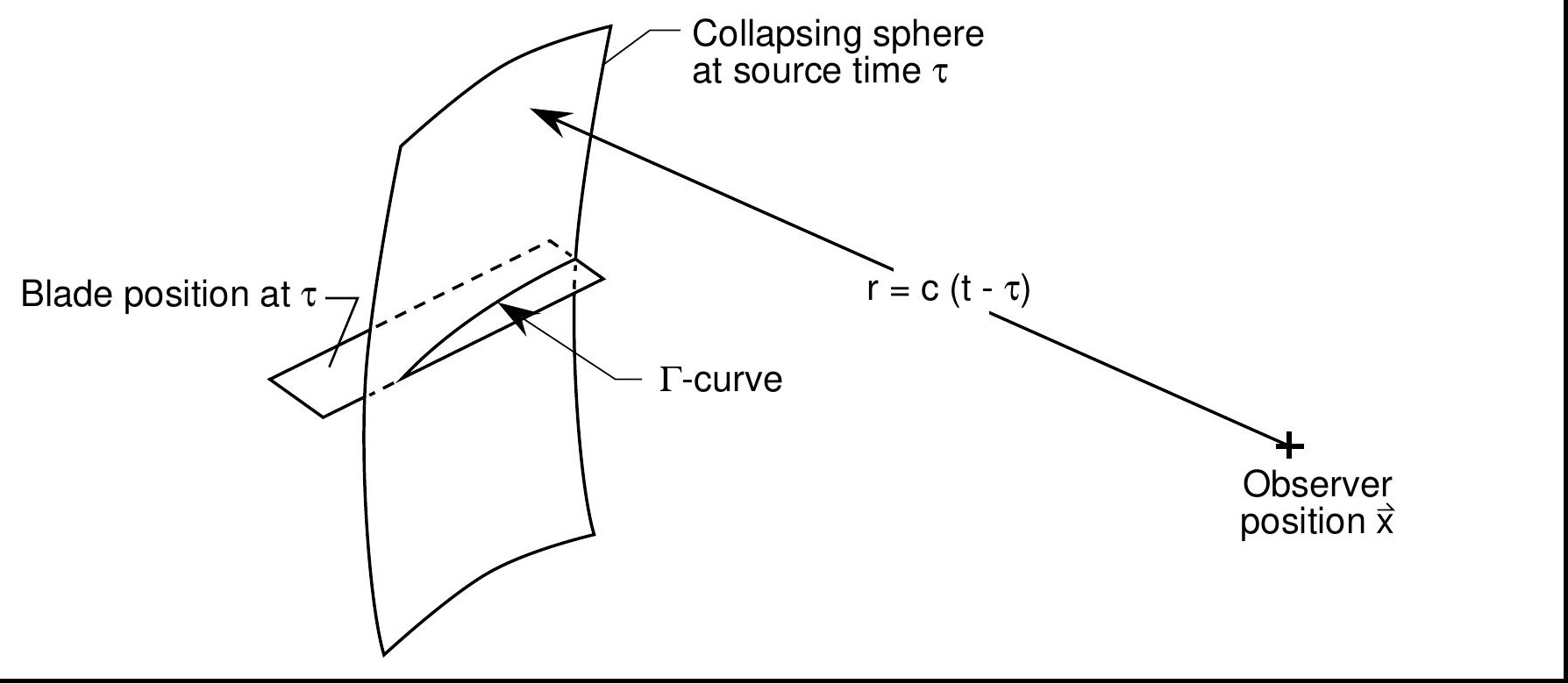


Observer position

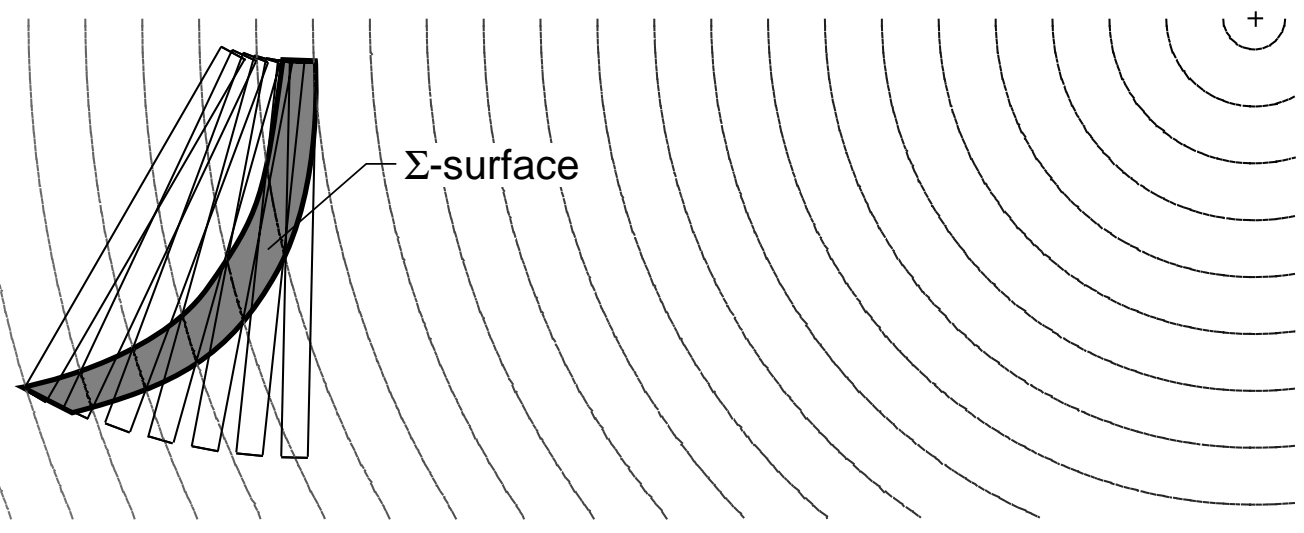

